\title{
A Refined QSO Selection Method Using Diagnostics
}

\author{
Dae-Won Kim ${ }^{1,2,3}$, Pavlos Protopapas ${ }^{1,3}$, Markos Trichas ${ }^{1}$, \\ Michael Rowan-Robinson ${ }^{4}$, Roni Khardon ${ }^{5}$, Charles Alcock ${ }^{1}$ \\ and Yong-Ik Byun ${ }^{2,6}$ \\ ${ }^{1}$ Harvard-Smithsonian Center for Astrophysics, Cambridge, MA, USA \\ ${ }^{2}$ Department of Astronomy, Yonsei University, Seoul, South Korea \\ ${ }^{3}$ Institute for Applied Computational Science, Harvard University, Cambridge, MA, USA \\ ${ }^{4}$ Astrophysics Group, Imperial College, London, UK \\ ${ }^{5}$ Department of Computer Science, Tufts University, Medford, MA 02155, USA \\ ${ }^{6}$ Yonsei University Observatory, Yonsei University, Seoul, South Korea
}

\begin{abstract}
We present 663 QSO candidates in the Large Magellanic Cloud (LMC) that were selected using multiple diagnostics. We started with a set of 2,566 QSO candidates selected using the methodology presented in our previous work based on time variability of the MACHO LMC light curves. We then obtained additional information for the candidates by cross-matching them with the Spitzer SAGE, the 2MASS, the Chandra, the XMM, and an LMC UBVI catalogues. Using that information, we specified diagnostic features based on mid-IR colours, photometric redshifts using SED template fitting, and X-ray luminosities, in order to discriminate more highconfidence QSO candidates in the absence of spectral information. We then trained a one-class Support Vector Machine model using those diagnostics features. We applied the trained model to the original candidates, and finally selected 663 high-confidence QSO candidates. We crossmatched those 663 QSO candidates with 152 newly-confirmed QSOs and 275 non-QSOs in the LMC fields, and found that the false positive rate was less than $1 \%$.
\end{abstract}

Keywords. Magellanic Clouds, methods: data analysis, quasars: general

\section{Introduction}

In previous work (Kim et al. 2011a) we developed a QSO selection method using a supervised classification model trained on a set of variability features extracted from the MACHO light curves, and including a variety of variable stars, non-variable stars and QSOs. The trained model showed a high efficiency of $80 \%$ and a low false positive rate of $25 \%$. Using that method, we selected 2,566 QSO candidates from the light-curve database. We then developed and employed a decision procedure on the basis of diagnostics using (1) mid-IR colours, (2) photometric redshifts and (3) X-ray luminosities for those candidates in order to separate high confidence QSO candidates (hereinafter hc-QSOs). We thus chose in total 663 hc-QSOs out of 2,566. Those 663 candidates are very probably QSOs; if confirmed, they will increase the number of known QSOs in the MACHO LMC database by a factor of $\sim 12$.

\section{Selection Methods}

We selected 2,566 QSO candidates from the MACHO light-curve database using the QSO selection method developed by Kim et al. (2011a), and applied multiple diagnostics tests on them. 


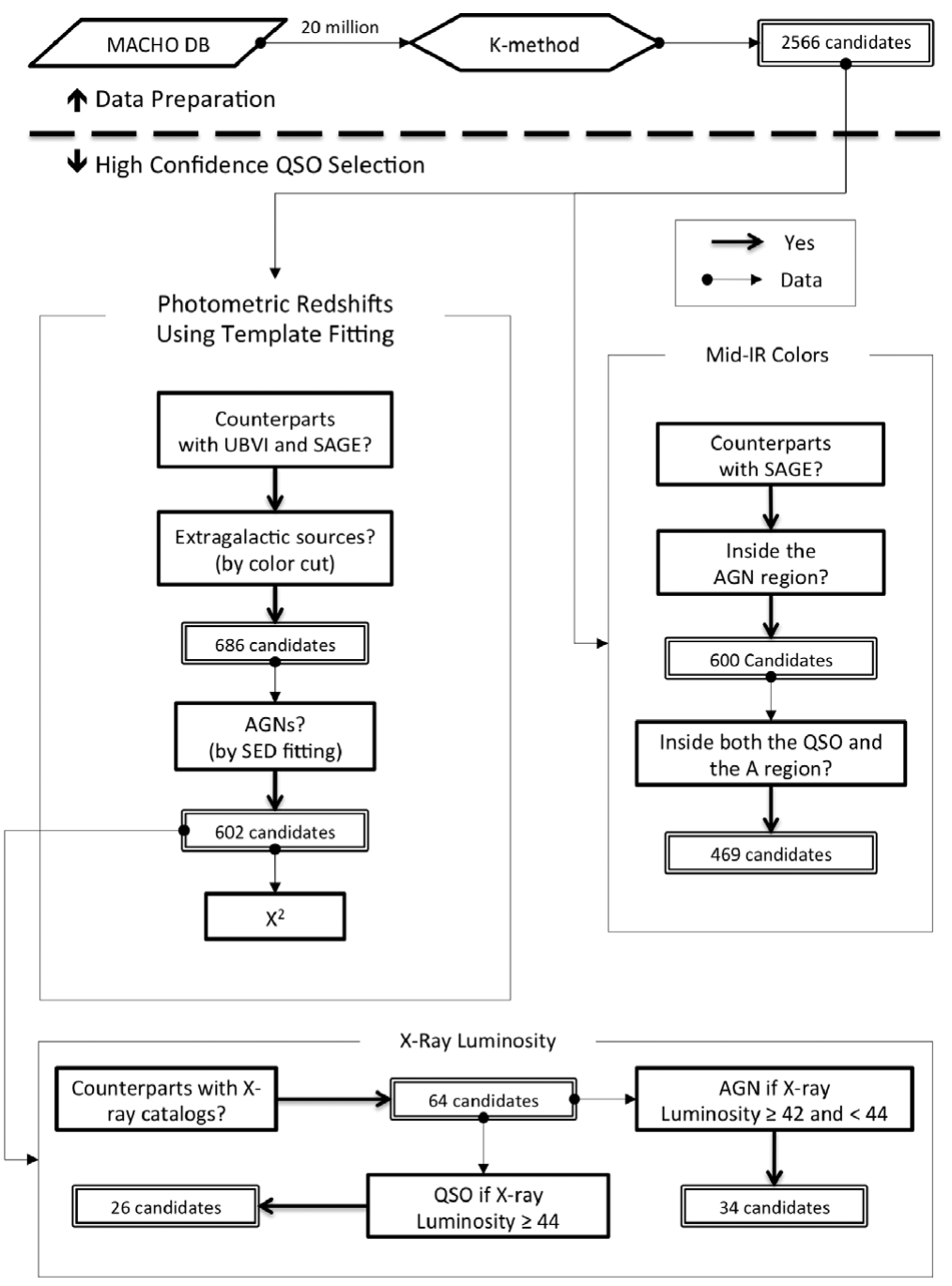

Figure 1. The rectangles with bold borderlines are the diagnostics. For most of the diagnostics, we determined whether the candidates are likely to be QSOs (solid line arrows). The thin arrows show the data flow. The double-lined rectangles show the number of candidates.

- Spitzer mid-IR properties. It is known that the mid-IR colour is an efficient discriminator between AGNs and stars/galaxies because their spectral energy distributions are substantially different from one another (Laurent et al. 2000; Lacy et al. 2004). Lacy et al. (2004) introduced a mid-IR colour cut to separate AGNs in the Spitzer SAGE catalogue (Surveying the Agents of a Galaxy's Evolution; Meixner et al. 2006). We used those mid-IR colour selections as the first diagnostic.

- Photometric redshifts using SED fitting. We cross-matched the 2,566 QSO candidates with the UBVI catalogue for the LMC (Zaritsky et al. 2004) and the 2MASS catalogue (Skrutskie et al. 2006), to extract $U B V I$ and $J H K$ magnitudes. We next separated stars from Galaxies plus AGNs (i.e., extragalatic sources) using a criterion proposed by Eisenhardt et al. (2004) and Rowan-Robinson et al. (2005). The 686 extragalatic sources thus identified were then fitted with galaxy templates in order to derive photometric redshifts (Rowan-Robinson et al. 2008). The template objects included three 
QSO, one starburst and 10 galaxies. The candidates which matched the QSO templates were considered to be QSOs.

- X-ray luminosities. We cross-matched the 2,566 QSO candidates with two X-ray point source catalogues, the Chandra X-ray source catalogue (Evans et al. 2010) and the XMM-Newton $2^{\text {nd }}$ Incremental Source catalogue (Watson et al. 2009). We found 88 counterparts. 64 of them fitted the SED templates and therefore had estimated photometric redshifts; we used those photometric redshifts and the X-ray fluxes from the catalogues to calculate their X-ray luminosities. The candidates showing high X-ray luminosity were deemed likely to be QSOs (Elvis et al. 1994; Persic et al. 2004).

We then employed the one-class Support Vector Machine (SVM) classification method to select just the high-confidence QSO candidates because we do not have negative examples (i.e., a non-QSO training set). To train a model, we define the diagnostics results as feature vectors. Fig. 1 outlines the calculation of the diagnostics, and the number of candidates for which the diagnostics are available. Kim et al. (2011b) give details about the selection method.

\section{Crossmatching with Newly Discovered QSOs by Kozłowski}

To estimate the efficiency and the false positive rate of our selection method, we crossmatched the 663 candidates with the 152 newly-discovered QSOs (Kozłowski et al. 2011) and 275 confirmed non-QSOs (i.e., false positives). We found that the yield was higher than $43 \%$, and the false positive rate was less than $1 \%$.

\section{Summary}

From 2,566 QSO candidates that were selected by the time variability of their MACHO light curves in the MACHO light-curve database, we used the methods described above to identify 663 high-confidence QSO candidates in the LMC fields. This set can be used as a target set for spectroscopic surveys as they should maximize the yield; that is important because spectroscopic observations for relatively faint objects such as the QSO candidates in dense- and wide-field areas around the LMC are extremely expensive in telescope time.

\section{References}

Eisenhardt, P. R., et al. 2004, ApJS, 154, 48

Elvis, M., et al. 1994, ApJS, 95, 1

Evans, I. N., et al. 2010, ApJS, 189, 37

Kim, D.-W., et al. 2011a, ApJ, 735, 68

Kim, D.-W., et al. 2011b, ArXiv e-prints

Kozłowski, S., et al. 2011, ArXiv e-prints

Lacy, M., et al. 2004, ApJS, 154, 166

Laurent, O., et al. 2000, A\& $A, 359,887$

Meixner, M., et al. 2006, AJ, 132, 2268

Persic M., et al. 2004, A\&AA, 419, 849

Rowan-Robinson, M., et al. 2008, MNRAS, 386, 697

Rowan-Robinson, M., et al. 2005, AJ, 129, 1183

Skrutskie, M. F., et al. 2006, AJ, 131, 1163

Watson, M. G., et al. 2009, A\&GA, 493, 339

Zaritsky, D., Harris J., Thompson, I. B., \& Grebel, E. K. 2004, AJ, 128, 1606 